\title{
Transcriptome analysis of Shank3- overexpressing mice reveals unique molecular changes in the hypothalamus
}

\author{
Chunmei Jin ${ }^{1,2 \dagger}$, Hyojin Kang ${ }^{3 \dagger}$, Shinhyun Kim ${ }^{1,2}$, Yinhua Zhang ${ }^{1,2}$, Yeunkum Lee ${ }^{1,2}$, Yoonhee Kim ${ }^{1,2}$ and \\ Kihoon $\operatorname{Han}^{1,2^{*}}$
}

\begin{abstract}
Various mutations in the SH3 and multiple ankyrin repeat domains 3 (SHANK3) gene are associated with neurodevelopmental and neuropsychiatric disorders. Thus far, synaptic abnormalities in multiple brain regions, including the hippocampus, prefrontal cortex, striatum, and ventral tegmental area, have been investigated in several lines of Shank3 mutant mice. However, although some reports have shown loss and gain of body weight in Shank3 knock-out and overexpressing transgenic (TG) mice, respectively, the potential functions of Shank3 in the hypothalamus, a brain region critically involved in energy intake and expenditure, are unknown. Hence, we first characterized endogenous Shank3 mRNA and protein expression in the hypothalamus of adult wild-type mice. Thereafter, we performed transcriptome analysis (RNA-sequencing) in the hypothalamus of adult Shank3 TG mice which mildly overexpress Shank3 proteins. By comparing the 174 differentially expressed genes in the hypothalamus with those previously reported in the striatum and medial prefrontal cortex (MPFC) of Shank3 TG mice, we found that 159 were hypothalamus-specific while only 15 were also observed in either the striatum or mPFC. Furthermore, gene set enrichment analysis of the RNA-sequencing analysis revealed that ribosome-related genes were enriched especially in the up-regulated genes of Shank3 TG hypothalamus, which is in contrast to the results of the Shank3 TG striatum and mPFC analyses, where ribosome-related genes were enriched in the down-regulated genes. Beyond revealing endogenous Shank3 mRNA and protein expression in the hypothalamus, our results suggest unique molecular changes in the hypothalamus of Shank3 TG mice compared with those in the striatum and mPFC.
\end{abstract}

Keywords: Shank3, Hypothalamus, Transcriptome

\section{Main text}

Deletions, duplications, and point mutations in the SH3 and multiple ankyrin repeat domains 3 (SHANK3) gene, encoding excitatory postsynaptic core scaffolding proteins, are causally associated with numerous neurodevelopmental and neuropsychiatric disorders. Several mouse lines of knock-out, knock-in, overexpression, and viral knockdown for the Shank3 gene have been generated and characterized, which has provided important insights into the neuronal pathophysiology of SHANK3-associated brain

\footnotetext{
* Correspondence: neurohan@korea.ac.kr

${ }^{+}$Chunmei Jin and Hyojin Kang contributed equally to this work.

${ }^{1}$ Department of Neuroscience, College of Medicine, Korea University, 73, Inchon-ro, Seongbuk-gu, Seoul 02841, South Korea

${ }^{2}$ Department of Biomedical Sciences, College of Medicine, Korea University, Seoul 02841, South Korea

Full list of author information is available at the end of the article
}

disorders. Specifically, detailed biochemical and synaptic abnormalities in various brain regions of the mice, including the prefrontal cortex [1-3], hippocampus [4-6], striatum [7-10], and ventral tegmental area [11], have been investigated. The hypothalamus is a brain region critical for regulating energy intake and expenditure [12]. Notably, reduced body weight of a line of Shank3 knock-out mice was reported [13]. Moreover, Shank3 transgenic (Shank3 TG) mice which mildly overexpress Shank3 proteins (by approximately 50\%) showed increased body weight and food intake [5]. Therefore, it is conceivable that Shank3 may have some functions in the hypothalamus; however, this has not been investigated thus far. In the present study, we aimed to understand expression and molecular functions of Shank3 in the hypothalamus, by performing transcriptome (RNA-sequencing, RNA-seq) analysis in

(C) The Author(s). 2018 Open Access This article is distributed under the terms of the Creative Commons Attribution 4.0 International License (http://creativecommons.org/licenses/by/4.0/), which permits unrestricted use, distribution, and 

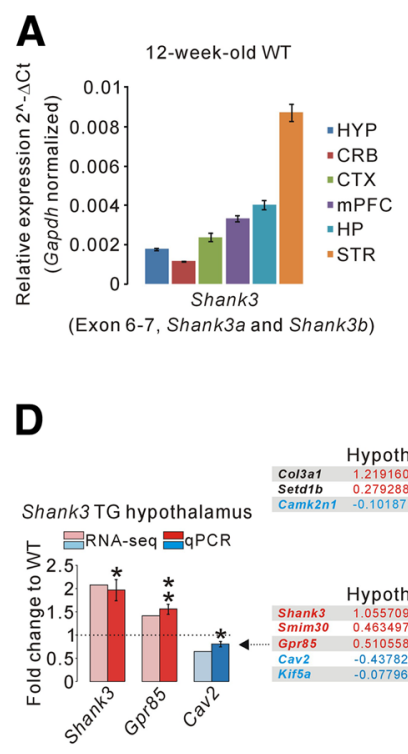

E gsea (Kegg List)

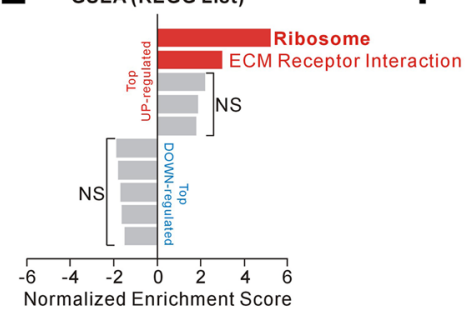

G

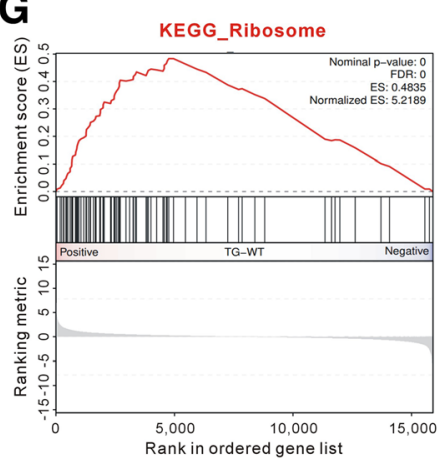

B

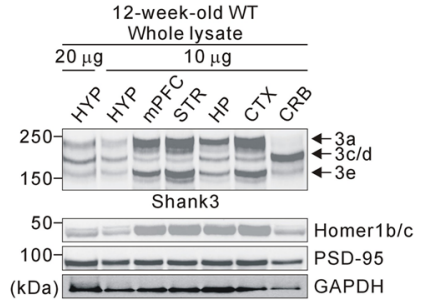

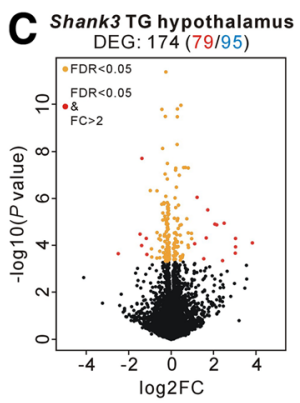

Shank3 TG DEGs col3a1 $\begin{aligned} & \text { Hypoth. Striatum mPFC H.219160 }-1.09303 \\ & 0.222854\end{aligned}$ Hypothalamus $174(79 / 95)$

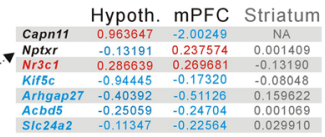

Striatum mPFC Hypoth. \begin{tabular}{lllll}
\hline Ehbp 1 & 0.542405 & -0.32767 & 0.039154
\end{tabular} $\begin{array}{llll}\text { Lama5 } & -0.43243 & 0.389623 & 0.220868 \\ \text { Ttr } & 4.090336 & 2.800976 & -0.44007 \\ \text { Adamts/4 } & -0.40039 & -0.52725 & 0.041494\end{array}$

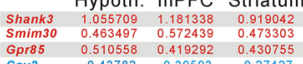
$\begin{array}{llll}\text { Gpr85 } & 0.510558 & 0.419292 & 0.430755 \\ \text { Cav2 } & -0.43782 & -0.39993 & -0.27427 \\ \text { Kif5a } & -0.07796 & -0.18033 & -0.17730\end{array}$

(1)

Striatum $75(33 / 42) \quad$ mPFC $195(82 / 113)$

$\mathbf{F}$

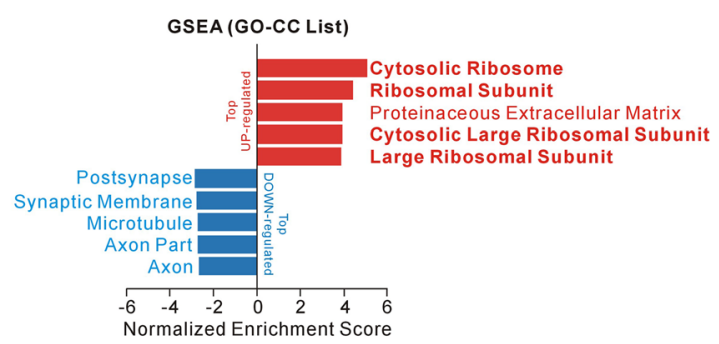

H

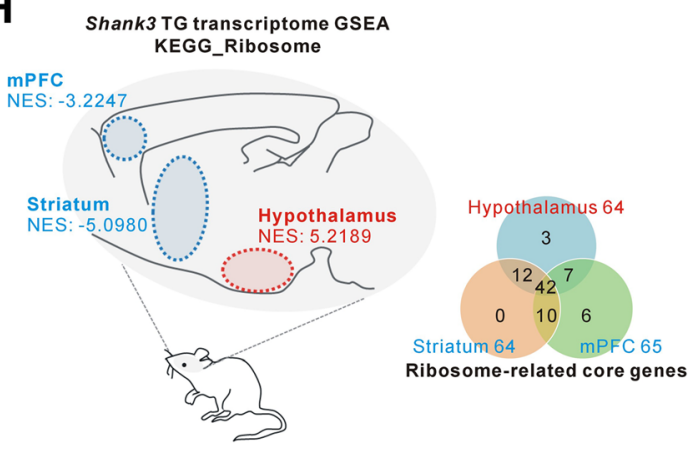

Fig. 1 (See legend on next page.) 
(See figure on previous page.)

Fig. 1 Characterization of Shank3 expression in the wild-type hypothalamus, and transcriptome analysis of the hypothalamus in Shank3 transgenic mice. a qRT-PCR results showing relative expression levels of Shank3 mRNA in various brain regions of adult wild-type (WT) mice. HYP, hypothalamus; CRB, cerebellum; CTX, cortex; HP, hippocampus; STR, striatum. b Western blot images showing relative expression levels of Shank3 (3a, 3c/d, 3e isoforms), Homer 1b/c, and PSD-95 proteins in various brain regions of adult WT mice. The amount of total proteins loaded in each well is indicated. $\mathbf{c}$ Volcano plot for the hypothalamic RNA-sequencing (RNA-seq) analysis of adult Shank3 TG mice. Differentially expressed genes (DEGs), defined by FDR $<0.05$, are shown as orange ( $F C<=2)$ and red $(F C>2)$ circles. FC, fold change. The complete lists of the RNA-seq analysis and DEGs are provided in Additional file 2: Tables 1 and 2. d The Venn diagram shows the numbers of common DEGs among the hypothalamus, striatum, medial prefrontal cortex (mPFC) of Shank3 TG mice. For the common DEGs, the log2FC values for each brain region are shown. The bar graph shows qRT-PCR results for Shank3, Gpr85, and Cav2 in the Shank3 TG hypothalamus. e, $\mathbf{f}$ The bar graphs show normalized enrichment scores (NESs) of the gene set enrichment analysis (GSEA) on the Kyoto Encyclopedia of Genes and Genomes (KEGG, E) and Gene Ontology-Cellular component (GO-CC, F) gene sets for the Shank3 TG hypothalamus RNA-seq analysis. Significant gene sets (FDR $<0.05)$ are highlighted in red and blue for up-regulated and down-regulated genes, respectively. The complete lists of the GSEA are provided in Additional file 2: Table 3. $\mathbf{g}$ The enrichment plot of RNA-seq analysis of Shank3 TG hypothalamus of the KEGG ribosome gene set. $\mathbf{h}$ The diagram shows opposite directional NES of the GSEA on KEGG ribosome gene set for the Shank3 TG hypothalamus compared with striatum and mPFC. Blue represents negative NES value (i.e., down-regulated genes), while red represents positive NES value (i.e., up-regulated genes). The Venn diagram shows the numbers of common ribosome-related core genes among the hypothalamus, striatum, and mPFC of Shank3 TG mice. The complete lists of the ribosome-related genes are provided in Additional file 2: Table 4. Data are presented as mean \pm SEM. ${ }^{*} P<0.05$ and ${ }^{* *} P<0.01$ (unpaired two-tailed Student's $t$-test)

the hypothalamus of adult (10 to 12-week old) Shank3 TG mice (Additional file 1).

We initially characterized the expression of endogenous Shank3 mRNA and protein in the hypothalamus because there was a report suggesting a lack of Shank3 expression in the hypothalamus [2]. In qRT-PCR experiments using primers targeting exon 6-7 of Shank3 (thus detecting Shank3a and Shank3b isoforms), Shank3 mRNA levels in the hypothalamus were relatively lower than those in the cortex, medial prefrontal cortex (mPFC), hippocampus, and striatum, but higher than those in the cerebellum of adult male wild-type (WT) mice (Fig. 1a). Consistent with the mRNA expression pattern, protein levels of Shank3 and its direct binding partner Homer $1 \mathrm{~b} / \mathrm{c}$ in the hypothalamus were relatively lower than those in other brain regions of adult male WT mice (Fig. 1b). Based on the expression of endogenous Shank3 in the hypothalamus, we next investigated molecular changes in the hypothalamus of Shank3 TG mice by performing a RNA-seq analysis of hypothalamic tissue from adult male WT and Shank3 TG mice (Additional file 2: Table 1). After applying adjusted $P$ values $(<0.05$, Benjamini-Hochberg correction) to the transcriptome analysis, we identified 174 differentially expressed genes (DEGs) (79 up-regulated and 95 down-regulated) in the Shank3 TG hypothalamus compared with the WT hypothalamus (Fig. 1c and Additional file 2: Table 2). Thereafter, we attempted to understand the specificity of the Shank3 TG hypothalamic DEGs, by comparing them to DEGs from two agematched Shank3 TG RNA-seq studies recently published by our group. One was from the striatum (75 DEGs; 33 up-regulated and 42 down-regulated) [14], and the other was from the medial prefrontal cortex (mPFC) (195 DEGs; 82 up-regulated and 113 down-regulated) of adult Shank3 TG mice [15]. We observed that the majority of DEGs found in each brain region were brain region-specific (Fig. 1d). Specifically, among the 174 DEGs of the Shank3 TG hypothalamus, 159 were hypothalamus-specific while only 15 were shared with either the striatum or MPFC. Notably, five genes were commonly altered in all three brain regions of Shank3 TG mice compared with those of the WT mice (Fig. 1d), among which we validated the changes of Shank3, G protein-coupled receptor 85 (Gpr85), and Caveolin 2 (Cav2) in the Shank3 TG hypothalamus by qRT-PCR experiments (Fig. 1d, left panel).

To understand representative biological pathways or functions of the 174 DEGs in the Shank3 TG hypothalamus, we performed Gene Ontology (GO) and Kyoto Encyclopedia of Genes and Genomes (KEGG) pathway analyses. However, we could not find significant terms in any category of the analyses, suggesting that the 174 DEGs may be too heterogeneous to be grouped into certain biological pathways or functions. Therefore, instead of focusing on the DEGs, we next performed gene set enrichment analysis (GSEA) of the RNA-seq analysis. GSEA is more useful to identify meaningful molecular signatures based on broader or overall expression changes in the transcriptome, regardless of fold change and statistical significance of each gene $[14,15]$. We applied three different groups of gene sets (Hallmark, KEGG, and GO gene sets) to the hypothalamic RNA-seq analysis. For the Hallmark gene sets, "Epithelial mesenchymal transition" was significantly represented by the up-regulated genes of the Shank3 TG hypothalamus (Additional file 2: Table 3). For the KEGG gene sets, "Ribosome" and "ECM receptor interaction" were enriched by the up-regulated genes of the Shank3 TG hypothalamus (Fig. 1e, g and Additional file 2: Table 3). Lastly, for the GO gene sets, "Cytosolic ribosome" and "Ribosomal subunit" were enriched by the up-regulated genes, while "Postsynapse" and "Synaptic membrane" were represented by the down-regulated genes of the Shank3 TG hypothalamus (Fig. If and Additional file 2: Table 3). Collectively, these results indicate that ribosome-related 
genes are enriched especially in the up-regulated genes of the Shank3 TG hypothalamus, which is reminiscent of our previous GSEA of the RNA-seq analyses of the striatum and mPFC of Shank3 TG mice [14, 15]. Notably, however, in the striatum and mPFC of Shank3 TG mice, ribosome-related genes were enriched in the down-regulated genes, but not in the up-regulated genes (Fig. 1h). Nevertheless, the ribosome-related core genes largely overlapped in all three brain regions (Fig. 1h, right panel and Additional file 2: Table 4), suggesting opposite directional changes of those genes in the hypothalamus compared with the striatum and mPFC of Shank3 TG mice. Nevertheless, it should be considered that the ribosomerelated genes in the Shank3 TG hypothalamus were not DEGs. Therefore, we consider that the GSEA results may reflect some, possibly ribosome-related, functional changes in the Shank3 TG hypothalamus which may lead to subtle, but overall, responses of ribosome-related genes. Our results show mRNA and protein expressions of Shank3 in the hypothalamus, and the effect of mild Shank3 overexpression on hypothalamic gene expression, thus providing a new platform to further investigate unique molecular and synaptic functions of Shank3 in the hypothalamus.

\section{Additional files}

Additional file 1: Materials and methods including information about mice, RNA sequencing and analysis, RNA purification and GRT-PCR, and biochemistry and antibodies for Western blotting. (DOCX 39 kb)

Additional file 2: Table S1. Summary of RNA-Seq mapping results (Shank3 TG hypothalamus 10-12 wk). Table S2. List of DEG (Differentially Expressed Gene) from RNA-Seq analysis results (Shank3 TG hypothalamus 10-12 wk). Table S3. GSEA (Gene-Set Enrichment analysis) (Shank3 TG hypothalamus 10-12 wk). Table S4. Comparison of ribosome-related GSEA core enrichment genes (Shank3 TG Hypothalamus, MPFC and Striatum). (XLSX $101 \mathrm{~kb})$

\section{Abbreviations}

Cav2: Caveolin 2; GO: Gene ontology; GPR85: G protein-coupled receptor 85; GSEA: Gene Set Enrichment Analysis; KEGG: Kyoto Encyclopedia of Genes and Genomes; mPFC: Medial prefrontal cortex; RNA-seq: RNA-sequencing; SHANK3: SH3 and multiple ankyrin repeat domains 3; TG: Transgenic

\section{Acknowledgements}

We thank the Laboratory Animal Research Center at Korea University College of Medicine for their animal care and support.

\section{Funding}

This work was supported by the National Research Foundation of Korea (NRF) grants funded by the Korea Government Ministry of Science and ICT (NRF2015M3C7A1028790, NRF-2018R1C1B6001235 and NRF-2018M3C7A1024603), by the Korea Institute of Science and Technology Information (K-18-L12-C08S01), and by Korea University Future Research Grant (K1824021).

\section{Availability of data and materials}

The datasets used and analyzed in the current study are available from the corresponding author on reasonable request. RNA-seq raw data were submitted to the GEO (Gene Expression Omnibus) repository under accession number GSE120609.

\section{Authors' contributions}

$\mathrm{CJ}, \mathrm{SK}, \mathrm{YZ}, \mathrm{YL}, \mathrm{YK}$ and $\mathrm{KH}$ designed and performed the experiments. HK and $\mathrm{KH}$ analyzed and interpreted the data. $\mathrm{KH}$ wrote the paper. All authors read and approved the manuscript.

\section{Ethics approval}

The WT and Shank3 TG mice were bred and maintained in a C57BL/6 J background according to the Korea University College of Medicine Research Requirements, and all the experimental procedures were approved by the Committees on Animal Research at the Korea University College of Medicine (KOREA-2016-0096).

\section{Consent for publication}

Not applicable.

\section{Competing interests}

The authors declare that they have no competing interests.

\section{Publisher's Note}

Springer Nature remains neutral with regard to jurisdictional claims in published maps and institutional affiliations.

\section{Author details}

'Department of Neuroscience, College of Medicine, Korea University, 73, Inchon-ro, Seongbuk-gu, Seoul 02841, South Korea. ${ }^{2}$ Department of Biomedical Sciences, College of Medicine, Korea University, Seoul 02841, South Korea. ${ }^{3}$ Division of National Supercomputing, KISTI, Daejeon 34141, South Korea.

Received: 1 October 2018 Accepted: 8 November 2018

Published online: 27 November 2018

References

1. Zhou Y, Kaiser T, Monteiro P, Zhang X, Van der Goes MS, Wang D, et al. Mice with Shank3 mutations associated with ASD and schizophrenia display both shared and distinct defects. Neuron. 2016;89(1):147-62. https://doi.org/ 10.1016/j.neuron.2015.11.023.

2. Lee J, Chung C, Ha S, Lee D, Kim DY, Kim H, et al. Shank3-mutant mice lacking exon 9 show altered excitation/inhibition balance, enhanced rearing, and spatial memory deficit. Front Cell Neurosci. 2015;9:94. https:// doi.org/10.3389/fncel.2015.00094.

3. Duffney L, Zhong P, Wei J, Matas E, Cheng J, Qin L, et al. Autism-like deficits in Shank3-deficient mice are rescued by targeting actin regulators. Cell Rep. 2015;11(9):1400-13. https://doi.org/10.1016/j.celrep.2015.04.064.

4. Kouser M, Speed HE, Dewey CM, Reimers JM, Widman AJ, Gupta N, et al. Loss of predominant Shank3 isoforms results in hippocampus-dependent impairments in behavior and synaptic transmission. J Neurosci. 2013;33(47): 18448-68. https://doi.org/10.1523/JNEUROSCI.3017-13.2013.

5. Han K, Holder JL Jr, Schaaf CP, Lu H, Chen H, Kang H, et al. SHANK3 overexpression causes manic-like behaviour with unique pharmacogenetic properties. Nature. 2013;503(7474):72-7. https://doi.org/10.1038/ nature12630.

6. Wang X, McCoy PA, Rodriguiz RM, Pan Y, Je HS, Roberts AC, et al. Synaptic dysfunction and abnormal behaviors in mice lacking major isoforms of Shank3. Hum Mol Genet. 2011;20(15):3093-108. https://doi.org/10.1093/ hmg/ddr212.

7. Peca J, Feliciano C, Ting JT, Wang W, Wells MF, Venkatraman TN, et al, Shank3 mutant mice display autistic-like behaviours and striatal dysfunction. Nature. 2011:472(7344):437-42. https://doi.org/10.1038/nature09965.

8. Bey AL, Wang X, Yan H, Kim N, Passman RL, Yang Y, et al. Brain regionspecific disruption of Shank3 in mice reveals a dissociation for cortical and striatal circuits in autism-related behaviors. Transl Psychiatry. 2018;8(1):94. https://doi.org/10.1038/s41398-018-0142-6.

9. Wang W, Li C, Chen Q, van der Goes MS, Hawrot J, Yao AY, et al. Striatopallidal dysfunction underlies repetitive behavior in Shank3-deficient model of autism. J Clin Invest. 2017;127(5):1978-90. https://doi.org/10.1172/ JCl87997.

10. Wang X, Bey AL, Katz BM, Badea A, Kim N, David LK, et al. Altered mGluR5Homer scaffolds and corticostriatal connectivity in a Shank3 complete knockout model of autism. Nat Commun. 2016;7:11459. https://doi.org/10. 1038/ncomms11459. 
11. Bariselli S, Tzanoulinou S, Glangetas C, Prevost-Solie C, Pucci L, Viguie J, et al. SHANK3 controls maturation of social reward circuits in the VTA. Nat Neurosci. 2016;19(7):926-34. https://doi.org/10.1038/nn.4319.

12. Timper K, Bruning JC. Hypothalamic circuits regulating appetite and energy homeostasis: pathways to obesity. Dis Model Mech. 2017;10(6):679-89. https://doi.org/10.1242/dmm.026609.

13. Drapeau E, Dorr NP, Elder GA, Buxbaum JD. Absence of strong strain effects in behavioral analyses of Shank3-deficient mice. Dis Model Mech. 2014;7(6): 667-81. https://doi.org/10.1242/dmm.013821.

14. Lee Y, Kim SG, Lee B, Zhang Y, Kim Y, Kim S, et al. Striatal transcriptome and Interactome analysis of Shank3-overexpressing mice reveals the connectivity between Shank3 and mTORC1 signaling. Front Mol Neurosci. 2017;10:201. https://doi.org/10.3389/fnmol.2017.00201.

15. Jin C, Kang H, Ryu JR, Kim S, Zhang Y, Lee Y, et al. Integrative brain transcriptome analysis reveals region-specific and broad molecular changes in Shank3-overexpressing mice. Front Mol Neurosci. 2018;11:250. https://doi. org/10.3389/fnmol.2018.00250.

Ready to submit your research? Choose BMC and benefit from:

- fast, convenient online submission

- thorough peer review by experienced researchers in your field

- rapid publication on acceptance

- support for research data, including large and complex data types

- gold Open Access which fosters wider collaboration and increased citations

- maximum visibility for your research: over $100 \mathrm{M}$ website views per year

At BMC, research is always in progress.

Learn more biomedcentral.com/submissions 